\title{
Fosfomycin trometamol and the single-dose treatment of cystitis
}

The launch of fosfomycin trometamol on the UK market for the single high-dose treatment of acute lower urinary tract infection introduces to these islands an old compound that was not previously available here. It also provides the latest example of the commercial exploitation of much truncated dosage regimens for simple cystitis, previous examples being the $2 \times 3-g$ sachet presentation of amoxycillin and the 3-day treatment packs of fluoroquinolones. A review of the agent and this therapeutic approach is, therefore, timely.

Fosfomycin is a naturally occurring antibiotic that was described originally under the name phosphonomycin in $1969 .{ }^{1}$ It has an unusually simple epoxypropylphosphonic acid structure ${ }^{2}$ and acts at a very early stage in bacterial cell wall synthesis by inhibiting the pyruvyl transferase enzyme that catalyses the condensation of phosphoenolpyruvate with $\mathrm{N}$-acetylglucosamine to form $\mathrm{N}$-acetylmuramic acid. ${ }^{3}$ Fosfomycin has a fairly broad spectrum of antibacterial activity, but the precise level of activity is difficult to assess; various constituents of culture media, including glucose, phosphates and sodium chloride, as well as $\mathrm{pH}$ and other test conditions, affect the activity, so that different results are obtained with different media. Lowest MIC values are observed generally with simple nutrient broth (or agar); MIC values are usually much higher in Mueller-Hinton medium. ${ }^{4-6}$ The susceptibility of some bacteria, notably Escherichia coli, is markedly enhanced in the presence of glucose-6phosphate, which is routinely added to media for tests of susceptibility to fosfomycin. The potentiation occurs because, in E. coli and some other gramnegative bacilli, glucose-6-phosphate induces a hexosephosphate transport pathway that can be used by fosfomycin to greatly improve access to the intracellular target. ${ }^{3}$ Mutations affecting this uptake system, which can be easily selected in vitro, abolish potentiation by glucose-6-phosphate and confer relative resistance to fosfomycin. There is presumably little opportunity for this type of resistance to develop in vivo, since the selection pressure is minimal, but mutations in the constitutive $\alpha$-glycerophosphate pathway that the drug also uses may give rise to clinical resistance. These types of resistance involve chromosomal genes, but plasmid-mediated resistance associated with enzymic inactivation of fosfomycin has also been described ${ }^{7,8}$ and plasmids carrying the resistance trait are circulating in Spain and other countries where the drug has been used extensively. ${ }^{9}$
Until recently, fosfomycin was available only as the sodium salt (for injection) and the calcium salt (for oral use). The advantage of the new trometamol formulation is that it is very soluble in water and provides greatly improved bioavailability compared with the calcium salt. ${ }^{10}$ However, on a weight-forweight basis the trometamol salt has half the activity of the other derivatives since trometamol-also known as tromethamine, or tris- (hydroxymethyl) -aminomethane-which is antibacterially inactive, almost doubles the mol. wt.

After absorption, fosfomycin is excreted by the renal route, with the trometamol salt achieving peak concentrations in bladder urine of $c .2000 \mathrm{mg} / \mathrm{L}$ after the recommended single oral dose of $3 \mathrm{~g}$; the elimination half-life is about $4 \mathrm{~h}^{11}$ and concentrations sufficient to inhibit most urinary pathogens are maintained for about $48 \mathrm{~h}^{12}$

It has been clear since the work of Charlton and his colleagues ${ }^{13}$ that abbreviated 3-day regimens are as effective as traditional 10-day treatment in uncomplicated cystitis. Single high-dose therapy was popularised by Bailey in New Zealand in the $1970 \mathbf{s}^{14-16}$ although its antecedents were much earlier. ${ }^{17}$ The perceived benefits of this practice are that it not only provides reliable treatment at minimum cost and maximum convenience, but that it also helps to identify the patients who may require more intensive urological investigation, because they do not respond to minimal therapy. Single-dose therapy is also likely to be safer than conventional regimens and reduces the opportunity for the emergence of bacterial resistance.

To ask of an antimicrobial agent that it should clear infection in a single passage through the urinary tract is a stringent test of its efficacy. Consistent success can be expected only of agents with half-lives that are sufficiently long to sustain effective concentrations in bladder urine. Fosfomycin trometamol is acceptable in this respect, but so too are trimethoprim and the fluoroquinolones. Rapidly excreted agents, such as nitrofurantoin and the $\beta$-lactam antibiotics are much less suitable, and use of massive doses is, contrary to popular belief, unlikely to much prolong the period for which inhibitory concentrations of the drug appear in bladder urine. ${ }^{18}$

Uncomplicated urinary tract infection is commonly overtreated and single high-dose therapy undoubtedly works in many cases. However, this therapeutic approach should not be seen as a universal panacea, even in infection that is confined to the lower urinary 
tract. ${ }^{19}$ The benefits of economy and convenience provided by single high-dose therapy also apply to treatment with a single conventional dose of any one of various oral agents each night for three nights. This approach also has the advantage of offering the patient

\section{References}

1. Hendlin D, Stapley EO, Jackson M et al. Phosphonomycin. a new antibiotic produced by strains of streptomyces. Science 1969: $166: 122-123$.

2. Christensen BG. Leanza WJ, Beattie TR et al. Phosphonomycin: structure and synthesis. Science 1969; 166: $123-125$.

3. Kahan FM, Kahan JS, Cassidy PJ, Kropp H. The mechanisms of action of fosfomycin (phosphonomycin). Ann NY Acad Sci $1974 ; 235: 364-386$.

4. Zimmerman SB. Stapley EO, Wallick H, Baldwin R. Phosphonomycin. IV. Susceptibility testing method and survey. In: Hobby GL (ed) Antimicrobial agents and chemotherapy - 1969. Bethesda, American Society for Microbiology. 1970: 303-309.

5. Haag R. Vömel W. Einflüsse auf die Aktivität von fosfomycin in vitro. Immunität und Infektion 1979; 7: 202-209.

6. Greenwood D, Jones A, Eley A. Factors influencing the activity of the trometamol salt of fosfomycin. Eur J Clin Microbiol $1986 ; 5: 29-34$.

7. Llaneza J, Villar CJ, Salas JA, Suarez JE, Mendoza MC, Hardisson C. Plasmid-mediated fosfomycin resistance is due to enzymic modification of the antibiotic. Antimicrob Agents Chemother 1985; 28: 163-164.

8. Arca P, Rico M, Brana AF, Villar CJ, Hardisson C, Suárez JE. Formation of an adduct between fosfomycin and glutathione: a new mechanism of antibiotic resistance in bacteria. Antimicrob Agents Chemother 1988; 21 : $1552-1556$.

9. Suárez JE, Mendoza MC. Plasmid-encoded fosfomycin resistance. Antimicrob Agents Chemother 1991; 35: 791-795. the reassurance of therapy that continues for the usual duration of the symptoms.

D. GREENWOOD

Department of Microbiology \& PHLS Laboratory, University Hospital, Queen's Medical Centre, Nottingham NG7 2UH

10. Ferrari V, Bonanomi L, Borgia M, Lodola E, Marca G. A new fosfomycin derivative with much improved bioavailability by oral route. Chemioterapia Antimicrobica $1981 ; 4: 59-63$.

11. Wilson P, Williams JD, Rolandi E. Comparative pharmacokinetics of fosfomycin trometamol, sodium fosfomycin and calcium fosfomycin in humans. In: Neu HC, Williams JD (eds) New trends in urinary tract infections. Basel, Karger. 1988: 136-142.

12. Segre G, Bianchi E, Cataldi A, Zannini G. Pharmacokinetic profile of fosfomycin trometamol (Monuril). Eur Urol 1987; 13 Suppl 1: 56-63.

13. Charlton CAC, Crowther A, Davies JG et al. Three-day and ten-day chemotherapy for urinary tract infections in general practice. $B M J 1976 ; 1: 124-126$

14. Bailey RR, Abbott GD. Treatment of urinary-tract infections with a single dose of amoxycillin. NZ Med J 1976;8: 324

15. Bailey RR, Abbott GD. Treatment of urinary-tract infection with a single dose of amoxycillin. Nephron 1977; 18: 316-320.

16. Bailey RR, Abbott GD. Treatment of urinary tract infection with a single dose of trimethoprim-sulphamethoxazole. Can Med Assoc J 1978; 118: 551-552.

17. Grüneberg RN, Brumfitt W. Single-dose treatment of acute urinary tract infection: a controlled trial. BMJ 1967; 3 649-651

18. Greenwood D, Kawada Y, O'Grady F. Treatment of acute bacterial cystitis: economy versus efficacy. Lancet 1980; 1 : 197.

19. Brumfitt W, Hamilton-Miller JMT. The optimal duration of antibiotic treatment of urinary infections. In: Neu HC Williams JD (eds) New trends in urinary tract infections. Basel, Karger. 1988: 62-77. 